\title{
Effects of Gravity and Inlet/Outlet Location on a Two-Phase Cocurrent Imbibition in Porous Media
}

\author{
M. F. El-Amin and Shuyu Sun \\ Computational Transport Phenomena Laboratory (CTPL), Physical Sciences and \\ Engineering Division (PSE), King Abdullah University of Science and Technology (KAUST), \\ Thuwal 23955-6900, Jeddah, Saudi Arabia \\ Correspondence should be addressed to M. F. El-Amin, mohamed.elamin@kaust.edu.sa
}

Received 15 December 2010; Accepted 8 January 2011

Academic Editor: Zhangxin Chen

Copyright (C) 2011 M. F. El-Amin and S. Sun. This is an open access article distributed under the Creative Commons Attribution License, which permits unrestricted use, distribution, and reproduction in any medium, provided the original work is properly cited.

\begin{abstract}
We introduce 2D numerical investigations of the problem of gravity and inlet/outlet location effects of water-oil two-phase cocurrent imbibition in a porous medium. Three different cases of side-, top-, and bottom-inlet location are considered. Two-dimensional computations are carried out using the finite element method. Intensive comparisons are done between considering and neglecting gravity effect on water saturation, pressures of water and oil as well as water velocity. Results are introduced either in curves or as $2 \mathrm{D}$ visualization graphs. The results indicate that the buoyancy effects due to gravity force take place depending on inlet location. So, the buoyancy force in the momentum equations of the co-current imbibition model cannot be neglected as done by several previous studies. Also, we note that the $2 \mathrm{D}$ zero gravity model has a uniform flow and may be represented as $1 \mathrm{D}$ flow unlike the 2D nonzero gravity model showing a nonuniform flow.
\end{abstract}

\section{Introduction}

Oil recovery by imbibition mechanism, from fractured reservoirs, is a significant research area in multiphase flow in porous media especially for water-flooding process in fractured oil reservoir. Fractured reservoirs are composed of the fracture network and matrix. Fractures have a high permeability and very low volume compared to the matrix, whose permeability is very low but it contains the majority of the oil. Water-flooding is used to increase oil recovery by increasing water pressure in fractures since water quickly surrounds oil saturated matrices of lower permeability. The water-flooding performance works well with the water-wet condition, and imbibition can lead to significant recoveries, while poor recoveries and early water breakthrough occur with oil-wet condition. Imbibition is defined as displacement of the nonwetting phase (oil) by the wetting phase (water) with dominant 
effect of the capillary force in porous media. Imbibition can occur in both countercurrent and cocurrent flow modes, depending on the fracture network and the water injection rates. In cocurrent imbibition, water pushes oil out of the matrix thus, both water and oil flows are in the same direction. Countercurrent imbibition is whereby a wetting phase imbibes into a porous matrix (rock), displacing the nonwetting phase out from one open boundary. Cocurrent imbibition is faster and more efficient than countercurrent imbibition. Countercurrent imbibition is often the only possible displacement mechanism for cases where a region of the matrix is completely surrounded by water in the fractures [1-5]. Imbibition has been investigated by many more authors either for cocurrent or countercurrent flows or both of them together [6-9]. Reis and Cil [10] introduced a one-dimensional model for oil expulsion by countercurrent water imbibition in rocks. Scaling of time imbibition was estimated by many of authors in terms of fluid and rock properties (e.g., see [11, 12]) while, recently, El-Amin and Sun [13] suggested a scaling law in terms of characteristic velocity (e.g., injection velocity), which may be useful for water-flooding mechanisms. Morrow and Mason [14] introduced a comprehensive review on recovery of oil by spontaneous imbibition. Kashchiev and Firoozabadi [15] gave analytical solutions for 1D countercurrent imbibition in water-wet media. Analytical analysis of oil recovery during countercurrent imbibition in strongly water-wet system was given by Tavassoli et al. [16]. The Barenblatt's model of spontaneous countercurrent imbibition was investigated by Silin and Patzek [17]. Behbahani et al. [18] have performed a simulation of countercurrent imbibition in water-wet fractured reservoirs.

In many of imbibition studies, authors have neglected the gravity force effect by dropping the gravity force term from the flow equations especially for the oil-water modeling. Wilkinson [19] studied the percolation model of immiscible displacement in the presence of buoyancy forces. Analytical and numerical solutions of gravity-imbibition and gravitydrainage processes were given by Bech et al. [20]. Tavassoli et al. [21] have introduced analysis of countercurrent imbibition with gravity in weakly water-wet system. A pore-scale study of gravity, capillary, and viscous forces during drainage in a two-dimensional porous medium was introduced by Løvoll et al. [22]. Effect of injection rate, initial water saturation and gravity on water injection in slightly water-wet fractured porous media was examined experimentally by Karimaie and Torsæter [23]. Ruth et al. [24] provided an approximate analytical solution for countercurrent spontaneous imbibition. The problems of buoyancydriven vertical migration of fluids have been treated analytically or numerically by some researchers such as, recently, Silin et al. [25] have introduced simple analytical solutions in a model of gas flow driven by a combination of buoyancy, viscous, and capillary forces of a problem of two-phase countercurrent fluid flow. Akin and Kovscek [26] studied spontaneous cocurrent water imbibition into diatomite samples (with low-permeability porous media) at various initial water saturations. Cai et al. [27] have considered the fractal characterization of spontaneous cocurrent imbibition in porous media. They introduced analytical expressions for characterizing a spontaneous cocurrent imbibition process of wetting fluid into gassaturated porous media proposed based on the fractal characters of porous media. Recently, El-Amin and Sun [28] introduced 1D/2D numerical investigations of the problem of gravity and inlet location effects of a water-oil two-phase countercurrent imbibition in a porous medium.

The purpose of this study is to investigate the influence of gravity acceleration for different locations of the inlet of a water-oil two-phase immiscible incompressible 2D flow cocurrent imbibition in a homogenous porous medium. It is assumed that the flow is combined capillary and buoyancy driven. 


\section{Basic Equations}

In this section, we introduce the basic equations of water-oil two-phase flow of immiscible fluids. We assume that flow is incompressible governed by the equations of mass conservation for each phase and the generalized Darcy's law, respectively, as:

$$
\begin{aligned}
& \frac{\partial\left(\varphi \rho_{\alpha} S_{\alpha}\right)}{\partial t}=-\nabla \cdot\left(\rho_{\alpha} u_{\alpha}\right)+q_{\alpha}, \quad \alpha=w, o, \\
& u_{\alpha}=-\frac{K k_{r \alpha}}{\mu_{\alpha}}\left(\nabla \cdot p_{\alpha}+\rho_{\alpha} g \nabla z\right), \quad \alpha=w, o .
\end{aligned}
$$

The index $\alpha$ denotes to the water (wetting-phase, $w$ ) and oil (nonwetting, $n w$ ), respectively. $S, p, q, u, k_{r}, \rho$, and $\mu$ are the phase saturation, pressure, mass flow rate, Darcy velocity, relative permeability, density and viscosity, respectively. The saturation $S_{\alpha}$ of the phases are constrained by,

$$
S_{w}+S_{o}=0
$$

One may defined the phase saturation as the fraction of the void volume of a porous medium filled by this fluid phase. The mass flow rate $q_{i \alpha}$, describe sources or sinks and can be neglected in the current study. The quantity

$$
k_{\alpha}=K k_{r \alpha}, \quad \alpha=w, o
$$

is known as effective permeability of the phase $\alpha$. The relative permeability of a phase is a dimensionless measure of the effective permeability of that phase. It is the ratio of the effective permeability of that phase to the absolute permeability. Also, it is interesting to define the quantities $m_{w}$ and $m_{o}$ which are known as mobility ratios of wetting and nonwetting phases, respectively, given by

$$
m_{\alpha}=\frac{k_{\alpha}}{\mu_{\alpha}}, \quad \alpha=w, o
$$

The normalized wetting phase saturation $S$ is given by,

$$
S=\frac{S_{w}-S_{i w}}{1-S_{o r}-S_{i w}}, \quad 0<S<1,
$$

where $S_{i w}$ is the irreducible (minimal) wetting phase saturation and $S_{o r}$ is the residual (minimal) nonwetting phase (oil) saturation. The expression of relation between the relative permeabilities and the normalized wetting phase saturation $S$ given as

$$
\begin{gathered}
k_{r w}=k_{r w}^{0} S^{a}, \\
k_{r o}=k_{r o}^{0}(1-S)^{b} .
\end{gathered}
$$


The empirical parameters $a$ and $b$ can be obtained from measured data either by optimizing to analytical interpretation of measured data, or by optimizing using a core flow numerical simulator to match the experiment. $k_{r w}^{0}=k_{r w}(S=1)$ is the endpoint relative permeability to water, and $k_{r o}^{0}=k_{r o}(S=0)$ is the endpoint relative permeability to the nonwetting phase.

The capillary pressure $p_{c}$ is defined as a difference between the nonwetting and wetting phase pressures,

$$
p_{c}=p_{o}-p_{w}
$$

the total velocity defined as

$$
u=u_{w}+u_{o}
$$

the total mobility is given by

$$
m=m_{w}+m_{o}
$$

the fractional flow functions are

$$
f_{w}(S)=\frac{m_{w}(S)}{m(S)}, \quad f_{o}(S)=\frac{m_{o}(S)}{m(S)}
$$

and the density difference is,

$$
\Delta \rho=\rho_{o}-\rho_{w}
$$

Using (2.1)-(2.2), and (2.7)-(2.10) with some mathematical manipulation one can find

$$
\frac{\partial\left(\varphi \rho_{w} S_{w}\right)}{\partial t}=-\nabla \cdot \rho_{w} f_{w}(S)\left\{K m_{o}(S)\left(\frac{d p_{c}}{d S_{w}} \nabla S_{w}-\Delta \rho g \nabla z\right)+u\right\}
$$

where $u$ is total velocity given by

$$
u=-K m(S)\left\{\nabla p-\left[\rho_{w} f_{w}(S)+\rho_{o} f_{o}(S)\right] g \nabla z\right\} .
$$

Alternatively, using (2.7), the governing equation (2.1) may be rewritten in the form

$$
\begin{gathered}
\frac{\partial\left(\varphi \rho_{w} S_{w}\right)}{\partial p_{c}}\left(\frac{\partial p_{o}}{\partial t}-\frac{\partial p_{w}}{\partial t}\right)=\nabla \cdot \rho_{w}\left[K m_{w}\left(\nabla p_{w}-\rho_{w} g \nabla z\right)\right] \\
\frac{\partial\left(\varphi \rho_{o}\left(1-S_{w}\right)\right)}{\partial p_{c}}\left(\frac{\partial p_{o}}{\partial t}-\frac{\partial p_{w}}{\partial t}\right)=\nabla \cdot \rho_{o}\left[K m_{o}\left(\nabla p_{o}-\rho_{o} g \nabla z\right)\right] .
\end{gathered}
$$


Both models, (2.12) and (2.14) are used intensively especially in the field of oil reservoir simulations.

The capillary pressure function is dependent on the pore geometry, fluid physical properties and phase saturations. The two phase capillary pressure can be expressed by Leverett dimensionless function $J(S)$ which is a function of the normalized saturation $S$,

$$
p_{c}=r\left(\frac{\varphi}{K}\right)^{1 / 2} J(S)
$$

The $J(S)$ function typically lies between two limiting (drainage and imbibition) curves which can be obtained experimentally. Correlation of the imbibition capillary pressure data depends on the type of application. For example, for water-oil system, [3, 15], the capillary pressure and the normalized wetting phase saturation are correlated as

$$
p_{c}=-B \ln S,
$$

where $B$ is the capillary pressure parameter, which is equivalent to $\gamma(\varphi / K)^{1 / 2}$, in the general form of the capillary pressure, (2.15), thus $B \equiv \gamma(\varphi / k)^{1 / 2}$, and $J(S) \equiv \ln S$. Note that $J(S)$ is a scalar nonnegative function.

The purpose of this section of study is to investigate the influence of gravity acceleration for different locations of the inlet of a two-phase immiscible incompressible flow in a homogenous porous medium for cocurrent imbibition.

It is assumed that the flow is combined capillary and buoyancy driven. Consider a rectangular core at irreducible water saturation, has inlet one face and outlet is on the opposite face, while all around surface except inlet and outlet is closed (cocurrent imbibition). The two-dimensional simplification case of the cylindrical core is a Cartesian rectangle of dimensions, $x=W, z=H$. Assuming that flow is incompressible and there is no mass transfer between the two phases. Under these assumptions the governing equations may be rewritten in the form

$$
\begin{gathered}
\varphi \frac{\partial S_{w}}{\partial p_{c}}\left(\frac{\partial p_{o}}{\partial t}-\frac{\partial p_{w}}{\partial t}\right)-\nabla \cdot\left[K m_{w}\left(\nabla p_{w}-\rho_{w} g \nabla z\right)\right]=0 \\
\varphi \frac{\partial S_{w}}{\partial p_{c}}\left(\frac{\partial p_{o}}{\partial t}-\frac{\partial p_{w}}{\partial t}\right)+\nabla \cdot\left[K m_{o}\left(\nabla p_{o}-\rho_{o} g \nabla z\right)\right]=0
\end{gathered}
$$

which is the combined Darcy's law and the equations of mass conservation for each phase in 2D. In order to consider a specific case of study we may use an empirical formula of the capillary pressure in terms of normalized saturation function. The capillary pressure and the normalized wetting phase saturation are correlated as in (2.16). 


\section{Initial and Boundary Conditions}

For the cocurrent imbibition in which the only open end is initially in contact with oil at ambient pressure, say, zero pressure (gauge pressure). The water pressure in the core is given by the capillary pressure relationship, (2.15)-(2.16), which at $t=0$ leads to

$$
\begin{gathered}
p_{o}=0, \quad t=0, \quad 0 \leq x \leq W, \quad 0 \leq z \leq H, \\
p_{w}=p_{o}-p_{c}\left(S_{i w}\right)=-p_{c}\left(S_{i w}\right), \quad t=0, \quad 0 \leq x \leq W, \quad 0 \leq z \leq H .
\end{gathered}
$$

It is worth mentioning that the equilibrium static pressure $p_{c}=\Delta \rho g z$ may be considered in the initial condition as well as $p_{c}=\Delta \rho g H$ in the bottom as a boundary condition. But it is negligible quantities because the domain is small-scale (we tested it), but it may has a significant effect for a large-scale simulation which will be the future work.

As the cocurrent imbibition process begins the oil in the open-end is replaced by water at the ambient pressure, so if one assumes zero capillary pressure at this open face. In this study we consider three different locations of the inlet and outlet, at side, top or bottom, namely, Cases 1, 2 and 3, as follows.

Case 1. Side inlet and opposite side outlet

$$
\begin{gathered}
p_{w}=p_{o}=0, \quad t>0, \quad x=0, \quad 0 \leq z \leq H \quad \text { (Inlet), } \\
q_{w}=q_{o}=0, \quad t>0, \quad 0 \leq x \leq W, \quad z=0, \\
q_{w}=q_{o}=0, \quad t>0, \quad 0 \leq x \leq W, \quad z=H, \\
q_{w}=p_{o}=0, \quad t>0, \quad x=W, \quad 0 \leq z \leq H \quad \text { (Outlet), }
\end{gathered}
$$

where $q_{w}$ and $q_{o}$ are the water and oil flow rate, respectively.

Case 2. Top inlet and bottom outlet

$$
\begin{gathered}
q_{w}=q_{o}=0, \quad t>0, \quad x=0, \quad 0 \leq z \leq H, \\
q_{w}=p_{o}=0, \quad t>0, \quad 0 \leq x \leq W, \quad z=0 \quad \text { (Outlet), } \\
p_{w}=p_{o}=0, \quad t>0, \quad 0 \leq x \leq W, \quad z=H \quad \text { (Inlet), } \\
q_{w}=q_{o}=0, \quad t>0, \quad x=W, \quad 0 \leq z \leq H .
\end{gathered}
$$

Case 3. Bottom inlet and top outlet

$$
\begin{gathered}
q_{w}=q_{o}=0, \quad t>0, \quad x=0, \quad 0 \leq z \leq H, \\
p_{w}=p_{o}=0, \quad t>0, \quad 0 \leq x \leq W, \quad z=0 \quad \text { (Inlet), } \\
q_{w}=p_{o}=0, \quad t>0, \quad 0 \leq x \leq W, \quad z=H \quad \text { (Outlet), } \\
q_{w}=q_{o}=0, \quad t>0, \quad x=W, \quad 0 \leq z \leq H .
\end{gathered}
$$



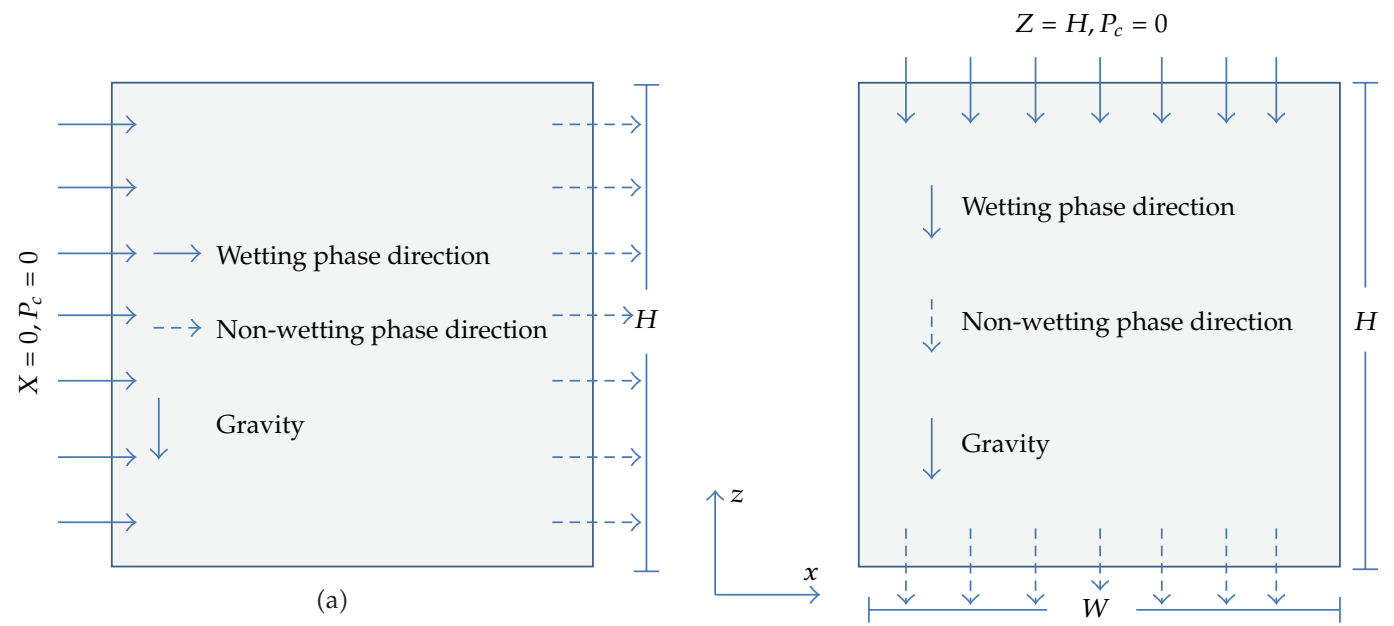

(b)

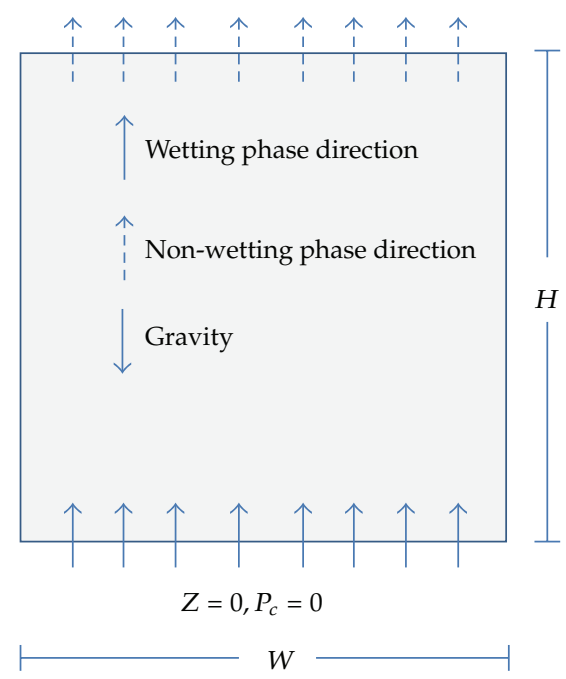

(c)

Figure 1: Schematic diagram of cocurrent imbibition for different inlet locations: (a) side, (b) top, and (c) bottom.

\section{Results and Discussion}

Figures 1(a), 1(b), and 1(c) show schematic diagrams of the problem of the two-phase cocurrent imbibition with gravity effect and different locations of the inlet (side, top and bottom) and the outlet is located opposite. Wetting phase imbibes inwards in a porous medium of height $H$ and width $W$ with zero capillary pressure at the inlet, and exits from the outlet at the opposite side and no-flow boundary at the other two boundaries.

Figures 2(a) and 2(b) show mesh distributions for Cases 1, 2, and 3, respectively, with a fine mesh on the inlet and outlet edges. Case 1 of a dimensional domain $(0.2,0.2) \mathrm{m}$ is meshed by 10439 nodes and 19968 triangle elements, corresponding to more than 81690 DOF (quadratic Lagrange elements), while Cases 2 and 3 of dimensional domain $(0.2,0.2) \mathrm{m}$ are meshed by 10591 nodes and 20272 triangle elements, corresponding to more than 82906 DOF 


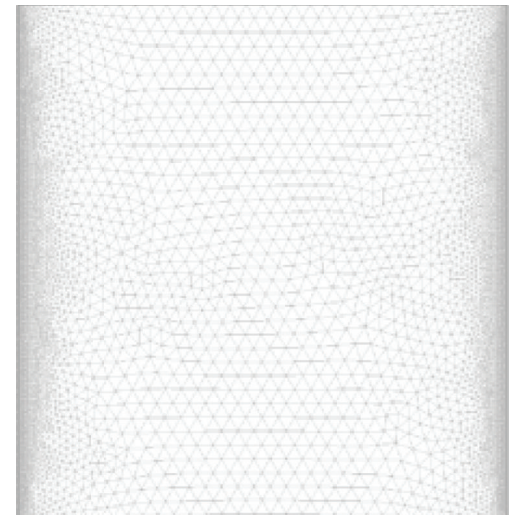

(a)

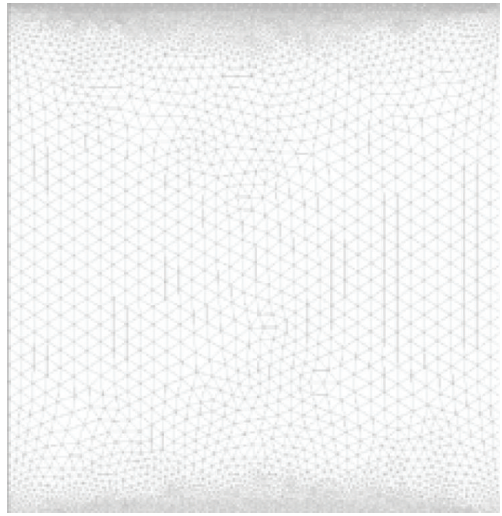

(b)

Figure 2: Mesh distribution for: (a) Case 1, (b) Cases 2 and 3.
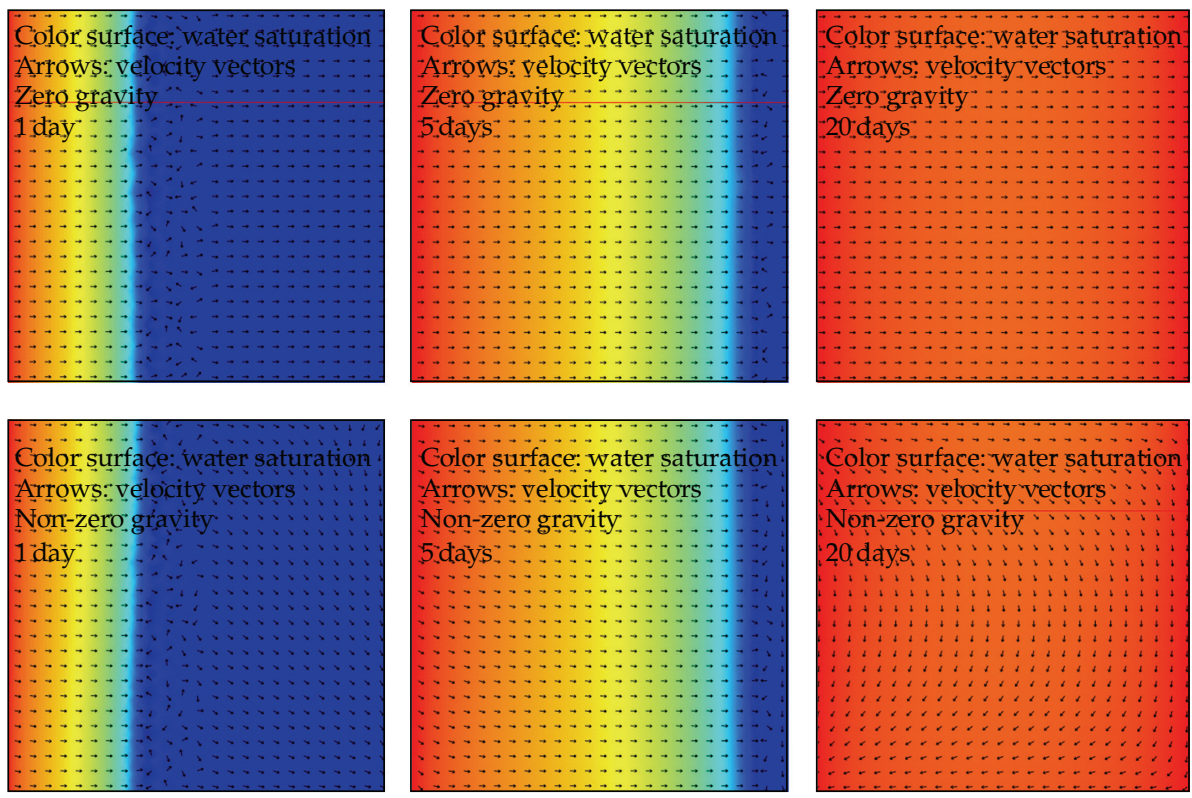

Figure 3: Distributions of water saturation and velocity vectors of zero, nonzero gravity of side-inlet case.

(quadratic Lagrange elements). All computations have been performed using the commercial software COMSOL version 3.5a with the direct solver UMFPACK, and were running on multi(8)-core workstation using SMP mode of parallel computation.

Figure 3 show distributions of water saturation and velocity vectors of zero, nonzero gravity of side-inlet case, at time imbibition of 1, 5, and 20 days. One can note that velocity vector of the zero-gravity case indicates has a uniform flow, while, the opposite is true for the case of nonzero gravity case shows a nonuniform flow.

Comparison between considering and neglecting gravity force on water saturation against $x$-axis of Case 1 (side-inlet), at $z=0.15 \mathrm{~m}$ is plotted in Figure 4 . We can note that there is a slight difference, of water saturation profile, between the considering and neglecting 


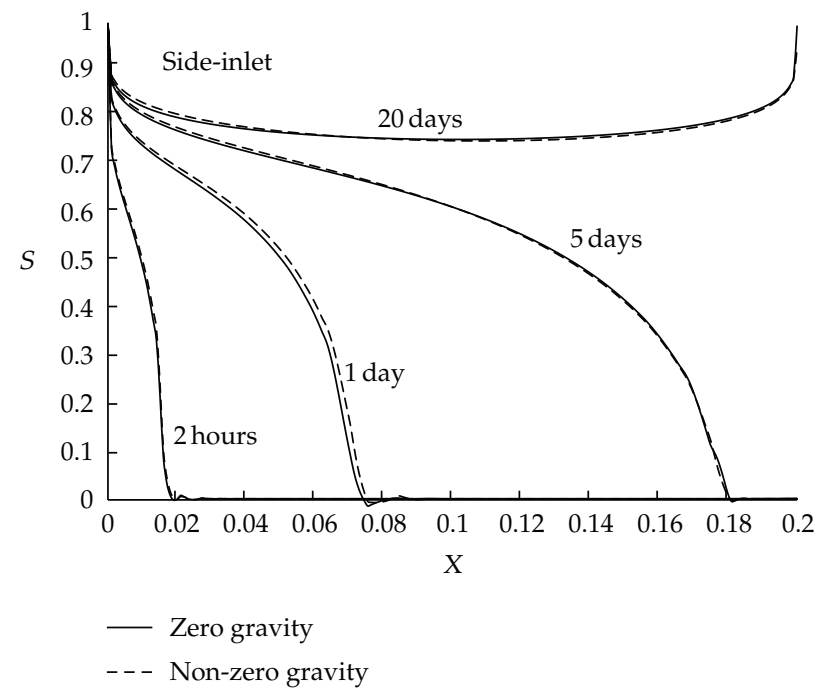

Figure 4: Comparison between considering and neglecting gravity effect on water saturation for the sideinlet case, at $z=0.15 \mathrm{~m}$.

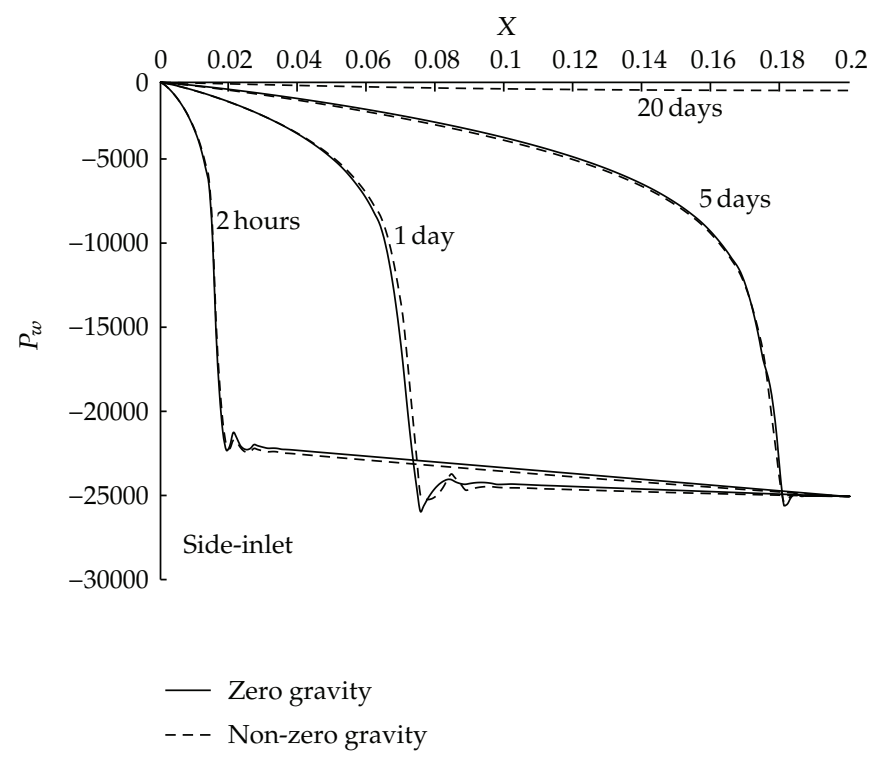

Figure 5: Comparison between considering and neglecting gravity effect on water pressure for the sideinlet case, at $z=0.15 \mathrm{~m}$.

of the gravity force on the model. Figure 5 shows a comparison between considering and neglecting gravity effect on water pressure against $x$-axis of Case 1 , at $z=0.15 \mathrm{~m}$. Also, the difference between zero and nonzero gravity cases is small, of the water pressure, especially for early imbibition time, while, the difference is a bit significant at big imbibition time (20 days). Comparisons between considering and neglecting gravity effect on oil pressure against $x$-axis of Case 1, at $z=0.15 \mathrm{~m}$, are plotted in Figure 6.

It is interesting to note a significant difference between zero and nonzero gravity cases, of oil pressure, at all imbibition time. It is clear that gravity force reduces the oil pressure and 


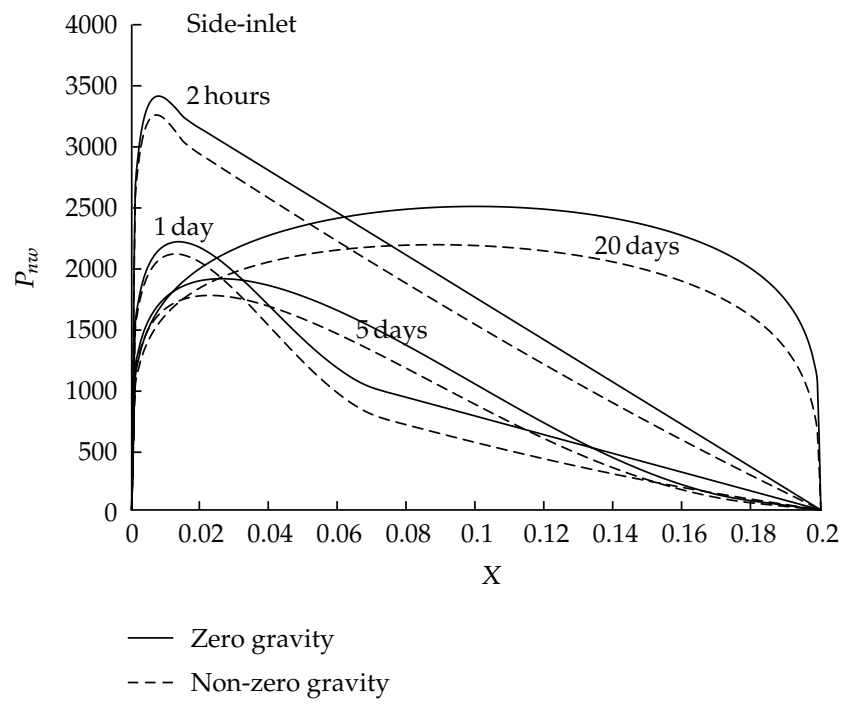

Figure 6: Comparison between considering and neglecting gravity effect on oil pressure for the side-inlet case, at $z=0.15 \mathrm{~m}$.

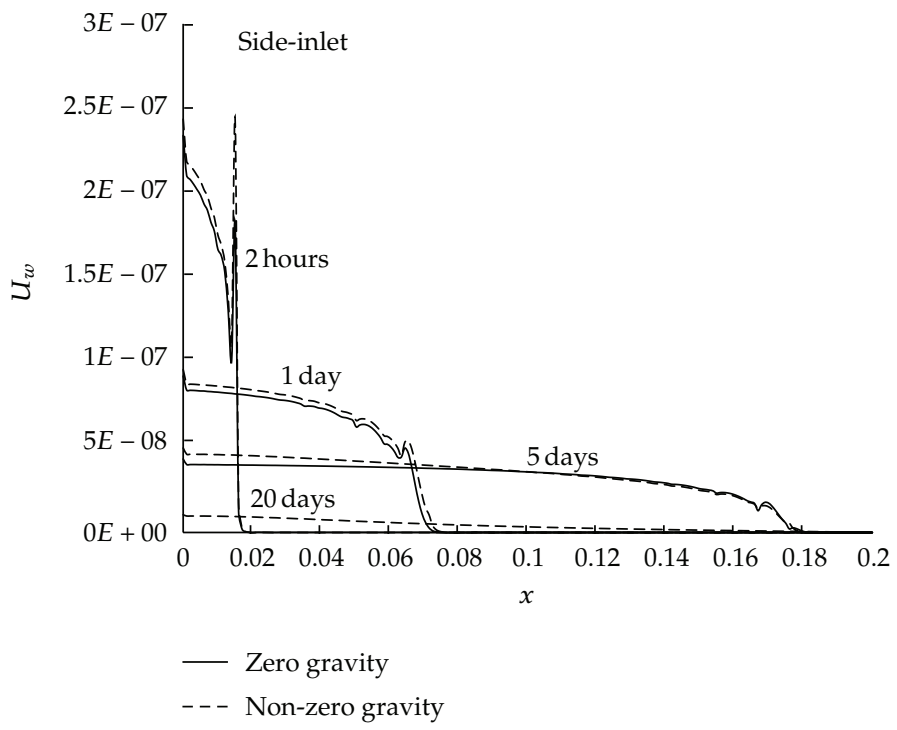

Figure 7: Comparison between considering and neglecting gravity effect on water $x$-velocity for the sideinlet case, at $z=0.15 \mathrm{~m}$.

consequently reduces the capillary pressure of the side-inlet case. In Figure 7, a comparison between considering and neglecting gravity effect on water $x$-velocity is plotted against $x$ axis of Case 1 , at $z=0.15 \mathrm{~m}$. From this figure, we may note a significant difference between the two cases especially for small $x$ (near to the inlet). This may be interpreted as the water imbibes inside medium pores the velocity is resisted by pores boundaries. Also, gravity has a significant effect after long imbibition time, so a quite big difference is noted at time 20 days between considering and neglecting gravity force. 

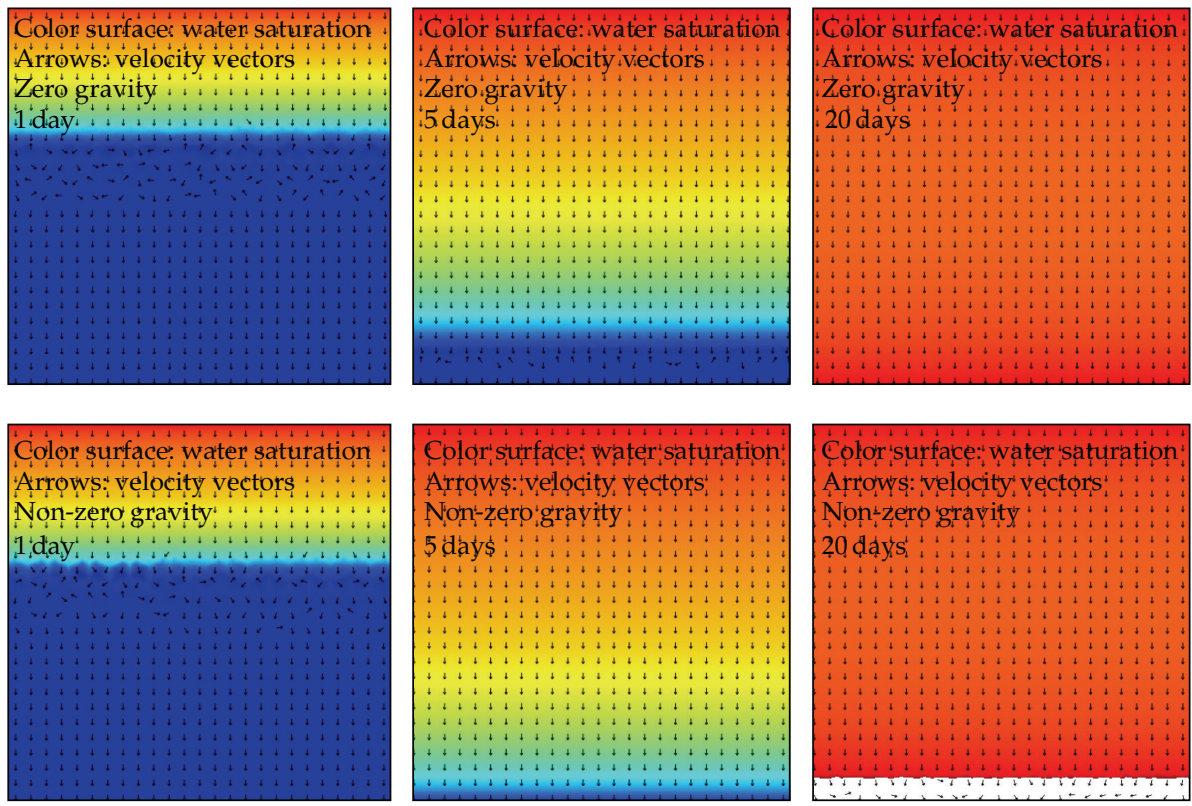

Figure 8: Distributions of water saturation and velocity vectors of zero, nonzero gravity for the top-inlet case.

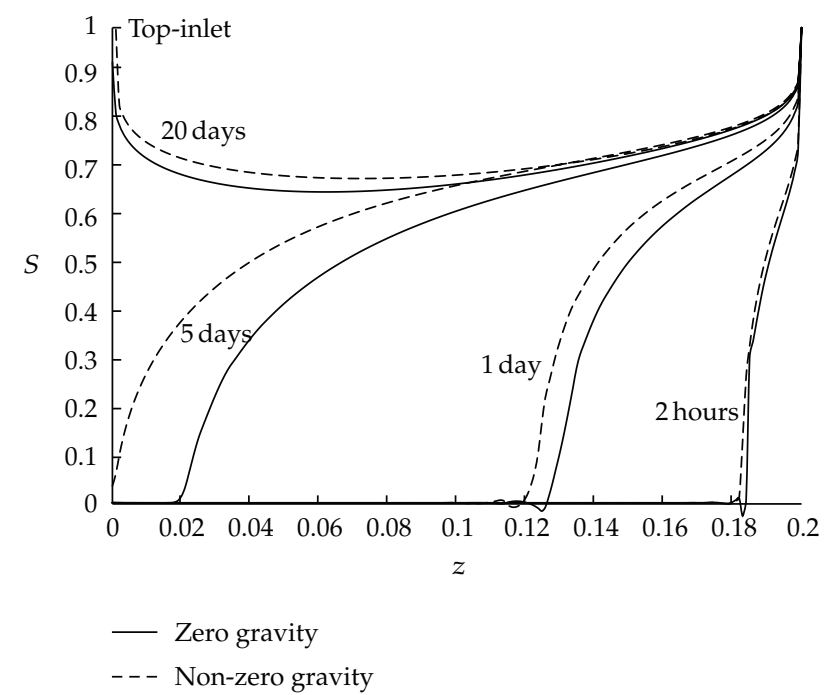

Figure 9: Comparison between considering and neglecting gravity effect on water saturation for the topinlet case, at $x=0.1 \mathrm{~m}$.

Figure 8 shows distributions of water saturation and velocity vectors of zero and nonzero gravity of top-inlet case, at time imbibition of 1, 5 and 20 days. From these figures we note that flow of the top-inlet case is uniform. Figure 9 illustrates a comparison between considering and neglecting gravity effect on water saturation against $z$-axis, at $x=0.1 \mathrm{~m}$ of top-inlet (Case 2).

It is interesting to note that the gravity force enhances water saturation for the topinlet case, thus, one can say that flow is gravity assisting. Water pressure profiles are plotted 


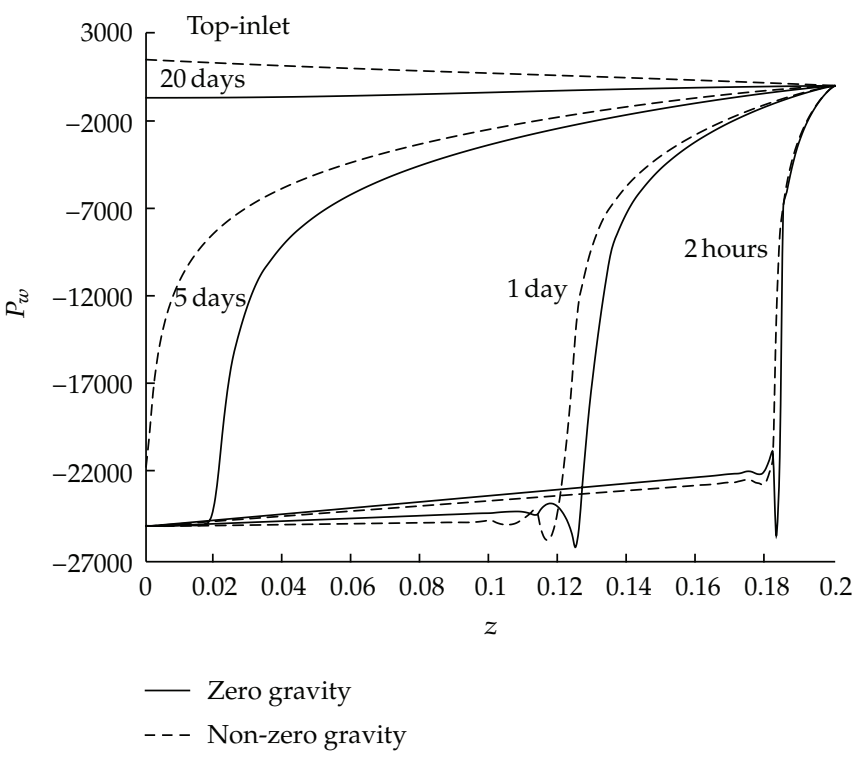

Figure 10: Comparison between considering and neglecting gravity effect on water pressure for the topinlet case, at $x=0.1 \mathrm{~m}$.

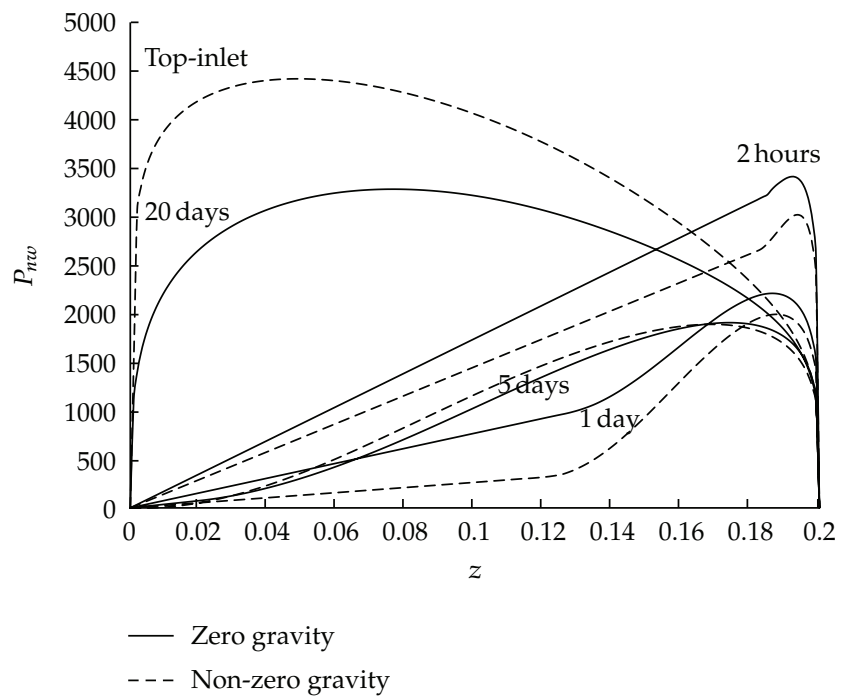

Figure 11: Comparison between considering and neglecting gravity effect on oil pressure for the top-inlet case, at $x=0.1 \mathrm{~m}$.

in Figure 10 for zero and nonzero gravity for the top-inlet case. It can be seen from this figure that the gravity force enhances water pressure for the top-inlet case and after a quite long imbibition time the water pressure becomes positive. The positive values of water pressure may be lead to the negative capillary pressure under certain conditions in a big depth. It is worth mentioning that the phenomenon of negative capillary pressure appeared in a number of experimental tests.

Comparison between considering and neglecting gravity effect on oil pressure against $z$-axis of Case 2, at $x=0.1 \mathrm{~m}$, is plotted in Figure 11. This figure indicates that the gravity 


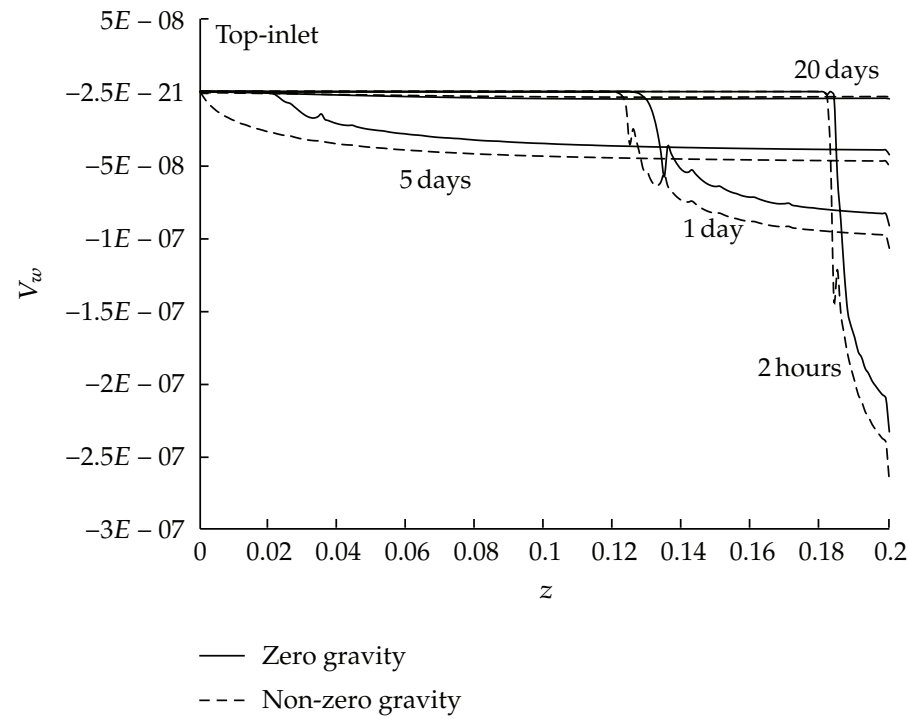

Figure 12: Comparison between considering and neglecting gravity effect on water $z$-velocity for the topinlet case, at $x=0.1 \mathrm{~m}$

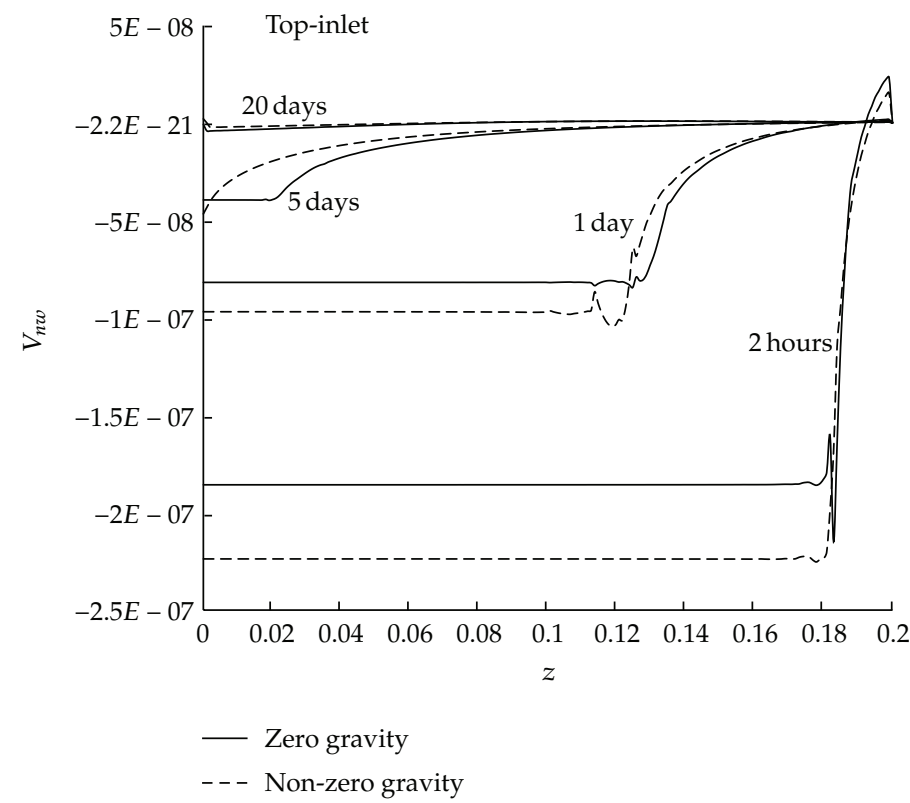

Figure 13: Comparison between considering and neglecting gravity effect on oil $z$-velocity for the top-inlet case, at $x=0.1 \mathrm{~m}$.

force has irregular effect on the oil pressure. In Figure 12 comparisons between considering and neglecting gravity effect on water $z$-velocity are plotted against $z$-axis of the top-inlet case. This figure shows a significant difference between the two cases especially for small time imbibition. Also, the oil z-velocity is plotted in Figure 13 against $z$-axis of the top-inlet case for zero and nonzero gravity. Also, we note a significant difference between the two cases for small time imbibition. 

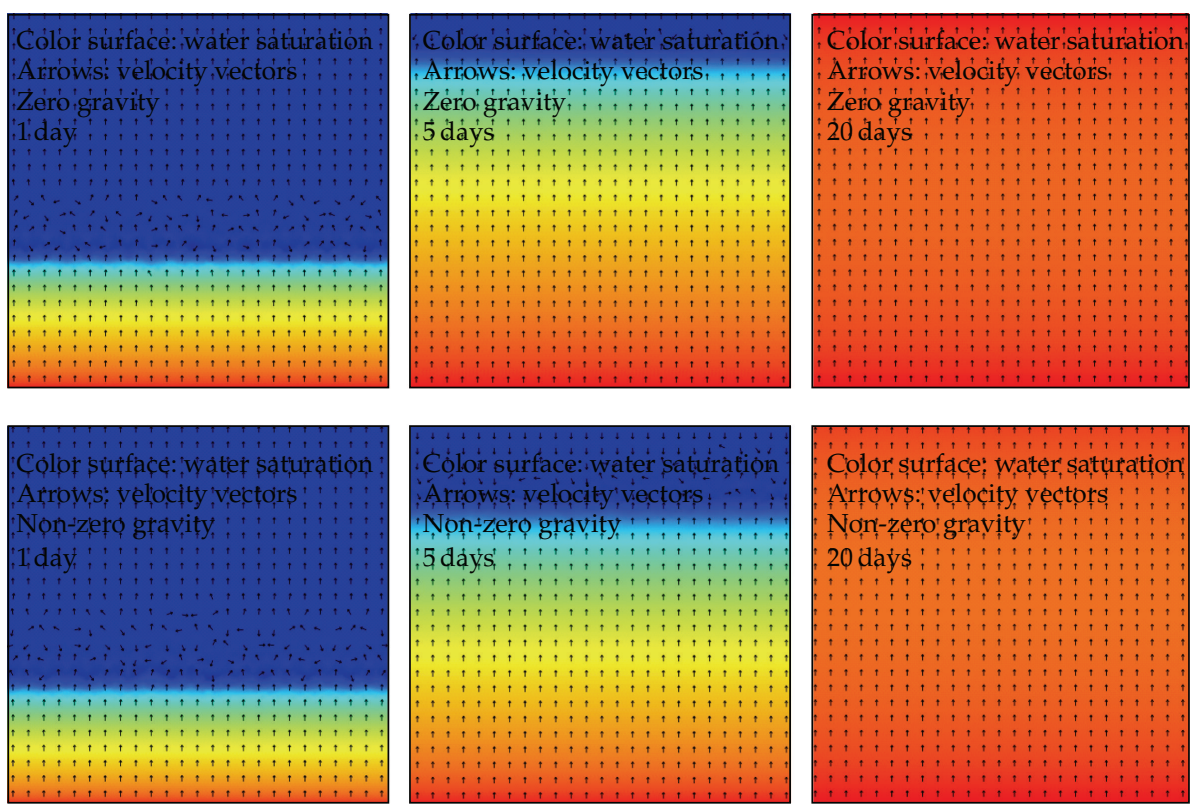

Figure 14: Distributions of water saturation and velocity vectors of zero, nonzero gravity for the bottominlet case.

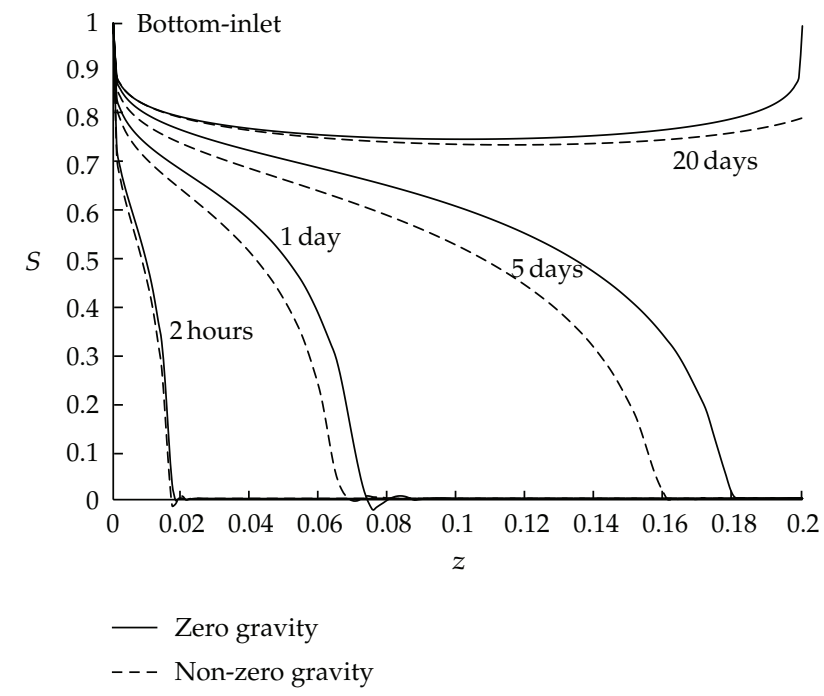

Figure 15: Comparison between considering and neglecting gravity effect on water saturation for the bottom-inlet case, at $x=0.1 \mathrm{~m}$.

Distributions of water saturation and velocity vectors for zero and nonzero gravity of bottom-inlet case, at time imbibition of 1, 5 and 20 days, are plotted in Figure 14. It can be seen from these figures we note that flow of the bottom-inlet case is uniform. In Figure 15 the water saturation profiles are plotted against $z$-axis, at $x=0.1 \mathrm{~m}$ of bottom-inlet case. It can be seen from this figure that gravity reduces water saturation for the bottom-inlet case, and the flow is gravity opposing. 


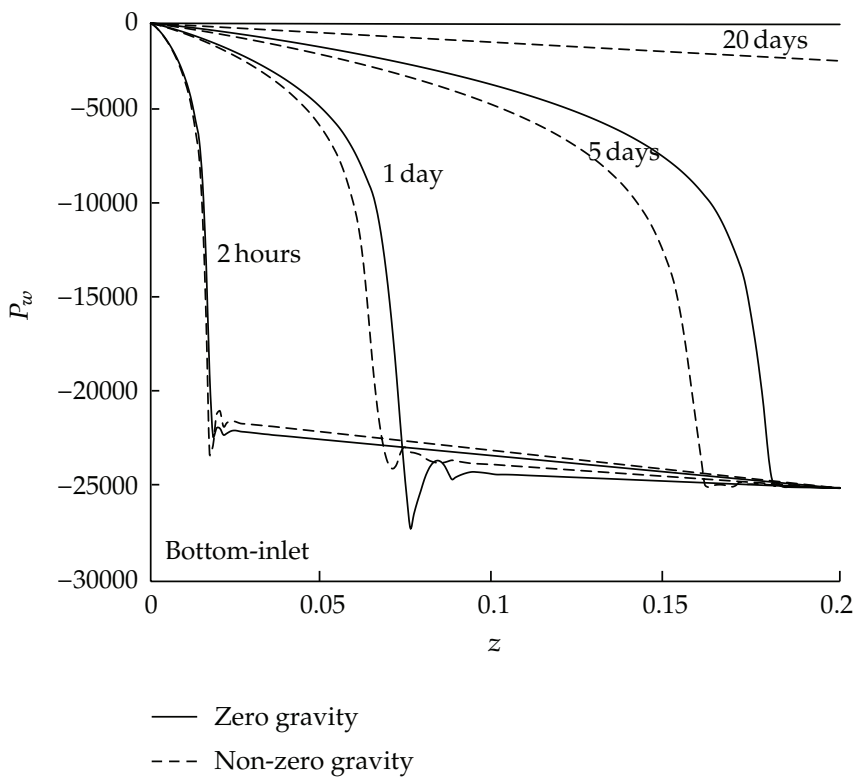

Figure 16: Comparison between considering and neglecting gravity effect on water pressure for the bottom-inlet case, at $x=0.1 \mathrm{~m}$.

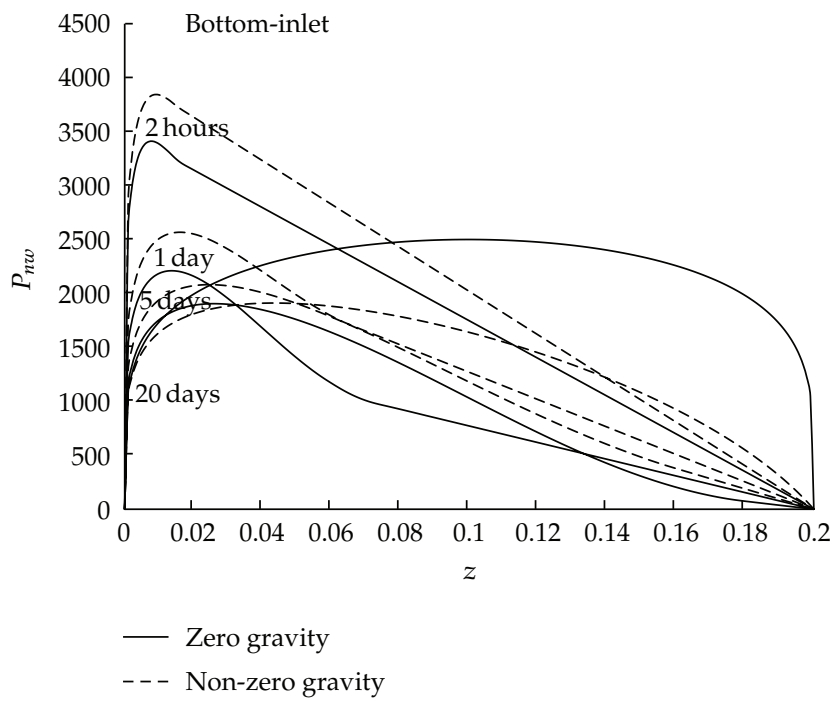

Figure 17: Comparison between considering and neglecting gravity effect on oil pressure for the bottominlet case, at $x=0.1 \mathrm{~m}$.

Figure 16 shows a comparison between considering and neglecting gravity effect on water pressure against $z$-axis of Case 3 , at $x=0.1 \mathrm{~m}$. This figure indicates that the gravity force reduces water pressure. Oil pressure profiles with zero and nonzero gravity are shown in Figure 17, for bottom-inlet case. Again, as the top-inlet case, the gravity force has irregular effect on the oil pressure for the bottom-inlet case. In Figure 18, water $z$-velocity is plotted 


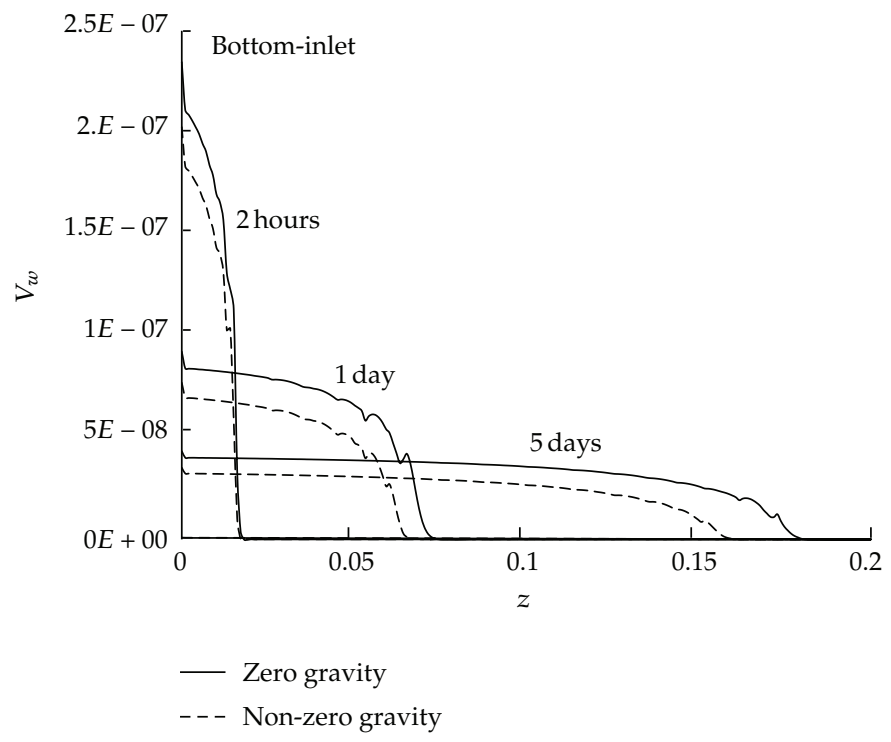

Figure 18: Comparison between considering and neglecting gravity effect on water $z$-velocity for the bottom-inlet case, at $x=0.1 \mathrm{~m}$.

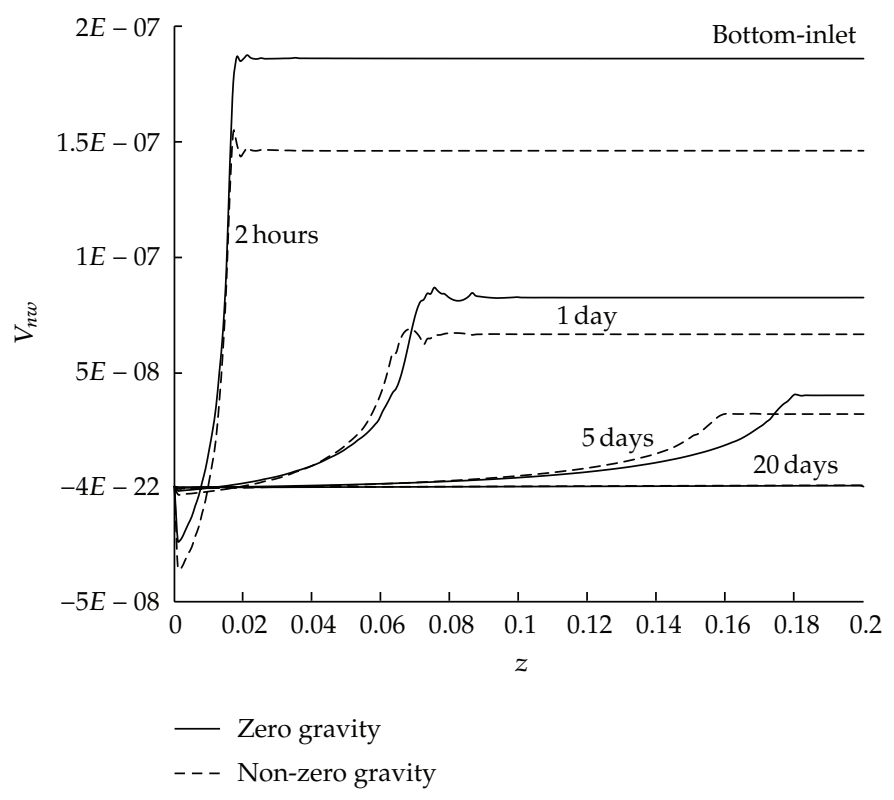

Figure 19: Comparison between considering and neglecting gravity effect on oil $z$-velocity for the bottominlet case, at $x=0.1 \mathrm{~m}$.

against $z$-axis of the bottom-inlet case, for zero and nonzero gravity. Also, the oil z-velocity is plotted in Figure 19 against $z$-axis of the bottom-inlet case for zero and nonzero gravity. Again, we note a significant difference between zero and nonzero gravity as shown in the two Figures 18 and 19. 


\section{Conclusions}

In this investigation we assure for including the gravity term in the mathematical model of two-phase cocurrent imbibition in a porous medium, and it cannot be ignored as done by several authors in the field. For this purpose we have considered three different cases of inlet location, namely, side, top and bottom. Numerical simulation was running of the water-oil system for maximum 20 days real imbibition time. Also, we have noted that the buoyancy effects due to gravity force take place depending on inlet/outlet location and a nonuniform 2D flow which is more realistic is detected. The current results will be useful for oil reservoir simulation and other similar simulation such as $\mathrm{CO}_{2}$ sequestration.

\section{References}

[1] B. J. Bourblaux and F. J. Kalaydjian, "Experimental study of cocurrent and countercurrent flows in natural porous media," SPE Reservoir Engineering, vol. 5, no. 3, pp. 361-368, 1990.

[2] M. E. Chimienti, S. N. Illiano, and H. L. Najurieta, "Influence of temperature and interfacial tension on spontaneous imbibition process," in Proceedings of the Latin American and Caribbean Petroleum Engineering Conference, vol. 53668, SPE, Caracas, Venezuela, April 1999.

[3] M. Pooladi-Darvish and A. Firoozabadi, "Cocurrent and countercurrent imbibition in a water-wet matrix block," SPE Journal, vol. 5, no. 1, pp. 3-11, 2000.

[4] H. L. Najurieta, N. Galacho, M. E. Chimienti, and S. N. Illiano, "Effects of temperature and interfacial tension in different production mechanisms," in Proceedings of the Latin American and Caribbean Petroleum Engineering Conference, vol. 69398, SPE, Buenos Aires, Argentina, 2001.

[5] G. Q. Tang and A. Firoozabadi, "Effect of pressure gradient and initial water saturation on water injection in water-wet and mixed-wet fractured porous media," SPE Reservoir Evaluation and Engineering, vol. 4, no. 6, pp. 516-524, 2001.

[6] R. W. Parsons and P. R. Chaney, "Imbibition model studies on water-wet carbonate rocks," SPE Journal, pp. 26-34, 1966.

[7] R. Iffly, D. C. Rousselet, and J. L. Vermeulen, "Fundamental study of imbibition in fissured oil fields," in Proceedings of the Annual Technical Conference, vol. 4102, SPE, Dallas, Tex, USA, 1972.

[8] G. Hamon and J. Vidal, "Scaling-up the capillary imbibition process from laboratory experiments on homogeneous samples," in Proceedings of the SPE European Petroleum Conferenc, vol. 15852, London, UK, October 1986.

[9] S. Al-Lawati and S. Saleh, "Oil recovery in fractured oil reservoirs by low IFT imbibition process," in Proceedings of the Annual Technical Conference and Exhibition, vol. 36688, SPE, Denver, Colo, USA, 1996.

[10] J. C. Reis and M. Cil, "A model for oil expulsion by counter-current water imbibition in rocks: onedimensional geometry," Journal of Petroleum Science and Engineering, vol. 10, no. 2, pp. 97-107, 1993.

[11] T. Babadagli, "Scaling of cocurrent and countercurrent capillary imbibition for surfactant and polymer injection in naturally fractured reservoirs," SPE Journal, vol. 6, no. 4, pp. 465-478, 2001.

[12] D. Zhou, L. Jia, J. Kamath, and A. R. Kovscek, "Scaling of counter-current imbibition processes in lowpermeability porous media," Journal of Petroleum Science and Engineering, vol. 33, no. 1-3, pp. 61-74, 2002.

[13] M. F. El-Amin and S. Sun, "Scaling of a two-phase countercurrent imbibition using characteristic velocity," Transport in Porous Media. Submitted.

[14] N. R. Morrow and G. Mason, "Recovery of oil by spontaneous imbibition," Current Opinion in Colloid and Interface Science, vol. 6, no. 4, pp. 321-337, 2001.

[15] D. Kashchiev and A. Firoozabadi, "Analytic solutions for 1D countercurrent imbibition in water-wet media," SPE Journal, vol. 8, no. 4, pp. 401-408, 2003.

[16] Z. Tavassoli, R. W. Zimmerman, and M. J. Blunt, "Analytic analysis for oil recovery during countercurrent imbibition in strongly water-wet systems," Transport in Porous Media, vol. 58, no. 1-2, pp. 173-189, 2005.

[17] D. Silin and T. Patzek, "On Barenblatt's model of spontaneous countercurrent imbibition," Transport in Porous Media, vol. 54, no. 3, pp. 297-322, 2004. 
[18] H. S. Behbahani, G. D. Donato, and M. J. Blunt, "Simulation of counter-current imbibition in waterwet fractured reservoirs," Journal of Petroleum Science and Engineering, vol. 50, no. 1, pp. 21-39, 2006.

[19] D. Wilkinson, "Percolation model of immiscible displacement in the presence of buoyancy forces," Physical Review A, vol. 30, no. 1, pp. 520-531, 1984.

[20] N. Bech, O. K. Jensen, and B. Nielsen, "Modeling of gravity-imbibition and gravity-drainage processes: analytic and numerical solutions," SPE Reservoir Engineering, vol. 6, no. 1, pp. 129-136, 1991.

[21] Z. Tavassoli, R. W. Zimmerman, and M. J. Blunt, “Analysis of counter-current imbibition with gravity in weakly water-wet systems," Journal of Petroleum Science and Engineering, vol. 48, no. 1-2, pp. 94-104, 2005.

[22] G. Løvoll, Y. Méheust, K. J. Måløy, E. Aker, and J. Schmittbuhl, “Competition of gravity, capillary and viscous forces during drainage in a two-dimensional porous medium, a pore scale study," Energy, vol. 30, no. 6, pp. 861-872, 2005.

[23] H. Karimaie and O. Torsæter, "Effect of injection rate, initial water saturation and gravity on water injection in slightly water-wet fractured porous media," Journal of Petroleum Science and Engineering, vol. 58, no. 1-2, pp. 293-308, 2007.

[24] D. W. Ruth, Y. Li, G. Mason, and N. R. Morrow, "An approximate analytical solution for countercurrent spontaneous imbibition," Transport in Porous Media, vol. 66, no. 3, pp. 373-390, 2007.

[25] D. Silin, T. Patzek, and S. M. Benson, "A model of buoyancy-driven two-phase countercurrent fluid flow," Transport in Porous Media, vol. 76, no. 3, pp. 449-469, 2009.

[26] S. Akin and A. R. Kovscek, "Imbibition studies of low-permeability porous media," in Proceedings of the Western Regional Meeting, vol. 54590, SPE, Anchorage, Ala, USA, May 1999.

[27] J. Cai, B. Yu, M. Zou, and L. Luo, "Fractal characterization of spontaneous co-current imbibition in porous media," Energy and Fuels, vol. 24, no. 3, pp. 1860-1867, 2010.

[28] M. F. El-Amin and S. Sun, "Effects of gravity and open-boundary location on a two-phase countercurrent imbibition in porous media," Journal of Porous Media. Submitted.

[29] Z. Chen, Reservoir Simulation: Mathematical Techniques in Oil Recovery, SIAM, Philadelphia, Pa, USA, 2007. 


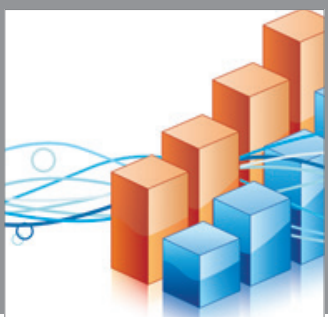

Advances in

Operations Research

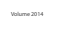

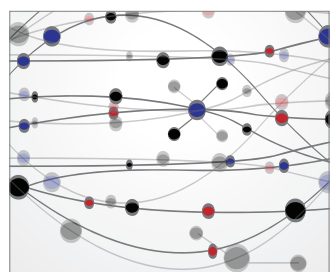

\section{The Scientific} World Journal
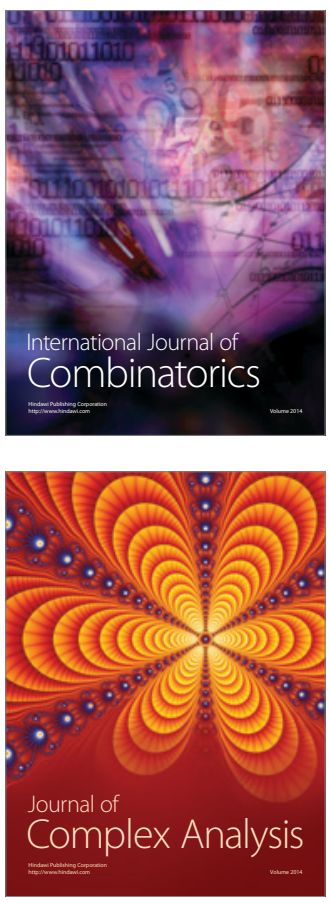

International Journal of

Mathematics and

Mathematical

Sciences
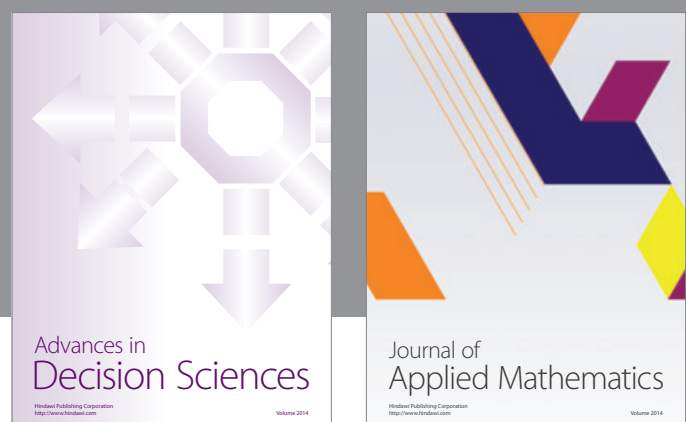

Journal of

Applied Mathematics
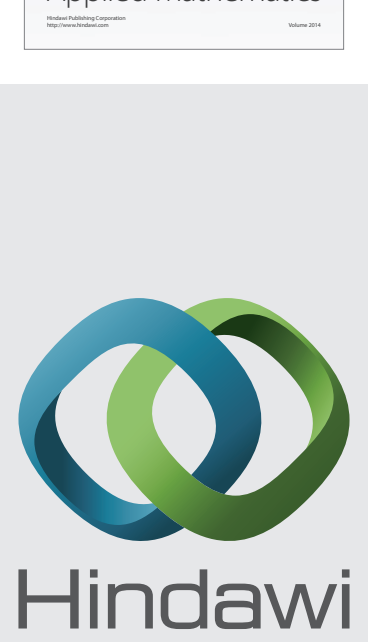

Submit your manuscripts at http://www.hindawi.com
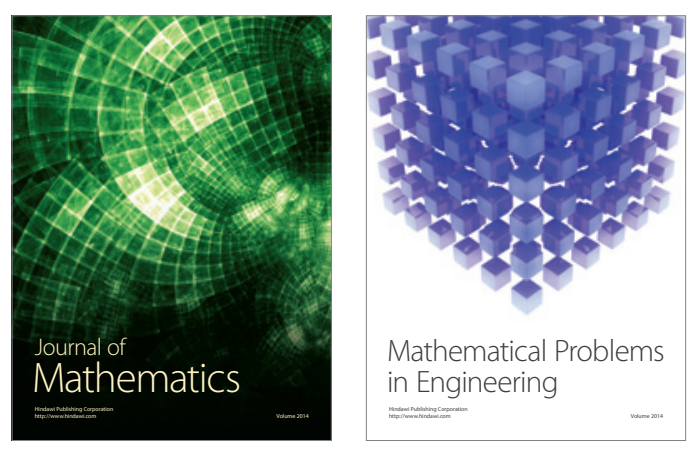

Mathematical Problems in Engineering
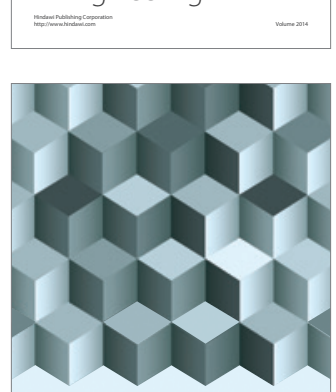

Journal of

Function Spaces
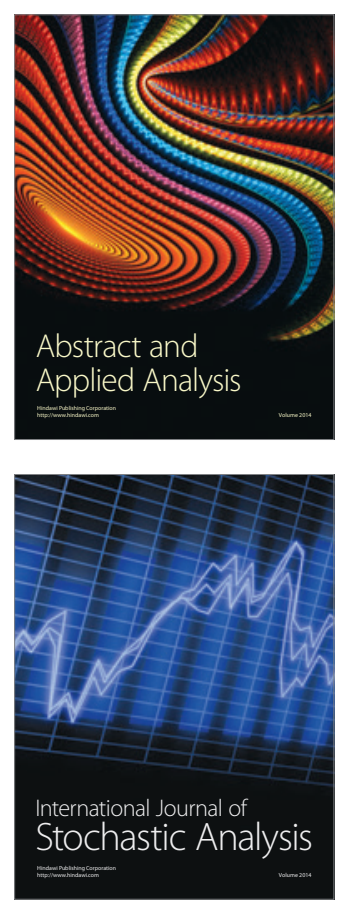

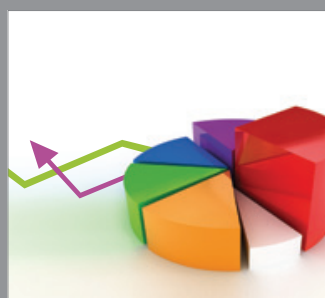

ournal of

Probability and Statistics

Promensencen
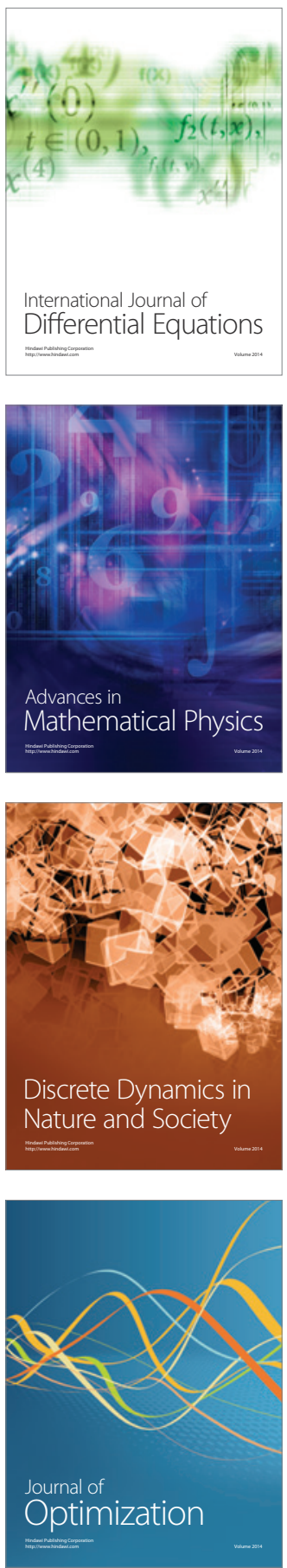